\section{Aids, Ethics and Public Policy}

Christine Pierce, Donald Van de Veer, editors, 241 pages, California, (price not yet decided) Wadsworth Inc, 1988.

This AIDS epidemic has raised many ethical issues. The editors of this book have assembled a collection of essays from various sources, including extracts from the popular press, legal journals and health insurance company reports. From these, the reader gains an overview of the general moral attitudes within our society and their legal consequences, in the context of the AIDS epidemic.

The book has been organised into four sections. The first, Perspectives, introduces some precepts of medical ethics and some basic facts about AIDS. The theme of the second section is the relationship between morality and the law. The third section, Law and Public Policy, discusses the feasibility of legal ways of containing the AIDS epidemic including quarantining of infected individuals and mass-population screening. The problems confronting health insurance companies are reviewed, particularly in the context of a country without a national health care system. It is of interest to the British reader to learn that some American States have introduced laws that forbid an HIV antibody test as a requirement for health insurance. The final section, Sexual Autonomy and the Constitution, (the American Constitution) looks at homosexuality and other sexual issues in relation to religion and the attitude of society.

The wide variation in style, which is inevitable with so many contributors, results in some chapters that are clear and well written while others are less good. Biographical details of the authors would have been of interest to those new to medical ethics, and would have lent weight to the arguments put forward.

There is a glossary of medical terminology but how could 'pneumocystis carinii pneumonia - a type of pneumonia primarily found in infants' get past the editors? Likewise a definition of epidemiology as 'a discipline concerned with tracking and discovering the cause(s) of an epidemic'? Although a dictionary definition, this is restricting and inaccurate in practical terms.

For all these criticisms, the editors did have a difficult task in trying to provide a text that could be an $\overline{\bar{\sigma}}$. introduction to AIDS and ethical issues? for some, yet function as a more $\overrightarrow{\vec{F}}$ specialist work for others. One $\mathcal{G}$ American review of this book suggests it should be required reading for medical students. Several chapters dwell too much on legal niceties and whilst this volume could be recommended to those with a particular interest many British 0 medical students would find it hard $\overrightarrow{0}$ going and a questionable addition to an already extensive reading list. For the $\vec{\omega}$ practising physician confronted with moral and ethical decisions in the management of patients with HIV infection this book does not raise any $\vec{A}$ new issues or resolve old dilemmas and $N$ as such may not be of much practical relevance. Definitely a book for those already involved in this complex subject but not one for those with a passing interest.

A EDWARDS

Lecturer, Honorary Senior Registrar in the Department of Genito-urinary Medicine, St Thomas's Hospital, London 